\title{
Dust Emission Modelling around a Stockpile by Using Computational Fluid Dynamics and Discrete Element Method
}

\author{
S.M. Derakhshani, D.L. Schott, G. Lodewijks \\ Section of Transport Engineering and Logistics, Department of Marine and Transport Technology, Faculty of \\ Mechanical, Maritime and Materials Engineering, Delft University of Technology, Mekelweg 2, 2628CD Delft, The
} Netherlands

\begin{abstract}
Dust emissions can have significant effects on the human health, environment and industry equipment. Understanding the dust generation process helps to select a suitable dust preventing approach and also is useful to evaluate the environmental impact of dust emission. To describe these processes, numerical methods such as Computational Fluid Dynamics (CFD) are widely used, however nowadays particle based methods like Discrete Element Method (DEM) allow researchers to model interaction between particles and fluid flow. In this study, air flow over a stockpile, dust emission, erosion and surface deformation of granular material in the form of stockpile are studied by using DEM and CFD as a coupled method. Two and three dimensional simulations are respectively developed for CFD and DEM methods to minimize CPU time. The standard $\kappa-\varepsilon$ turbulence model is used in a fully developed turbulent flow. The continuous gas phase and the discrete particle phase link to each other through gas-particle void fractions and momentum transfer. In addition to stockpile deformation, dust dispersion is studied and finally the accuracy of stockpile deformation results obtained by CFD-DEM modelling will be validated by the agreement with the existing experimental data.
\end{abstract}

Keywords: Dust emission, Stockpile, Computational fluid dynamics (CFD), Discrete element method (DEM).

PACS: 47.11.Df, 05.10.-a, 45.70.-n, 81.05.Rm

\section{INTRODUCTION}

Particle or dust emissions from sources such as unsealed surfaces and material stockpiles have an important effect on the human health, environment and industry equipment. Having a good understanding of dust liberation phenomenon can help to control and delimitate its harmful and undesired effects.

Several researchers have developed a multitude of techniques and methodologies to analyse the wind flow around the piles of open storage yards [1-3]. Computational Fluid Dynamic has largely implemented in the succeeding works to study the surrounding wind flow implications for the Aeolian particle transport in case of dune erosion[4]. Farimani et al. studied the dispersion of pollutants and dust release either from transportation or storage yards. Also some experiments and CFD simulations performed to investigate the role of saltation and creep module on the deformation and erosion rate of a sand pile[1,5].

In this study deformation and erosion of a sinusoidal sand pile will be studied by using coupled CFD and DEM methods.

\section{NOMENCLATURE}

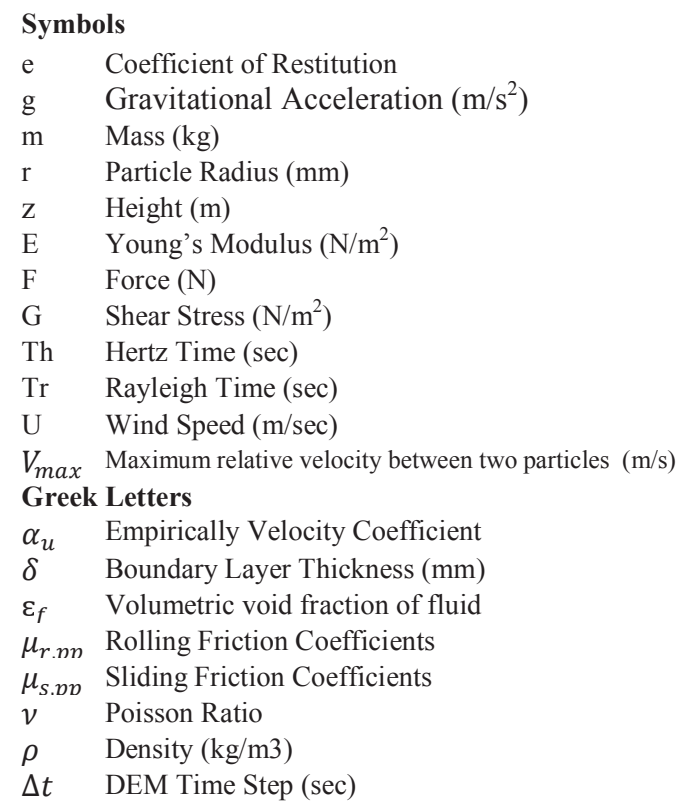




\section{COMPUTATIONAL MODEL}

In this section of the paper, the computational model and its assumptions and algorithms are explained and discussed.

\section{Discrete Element Method (DEM)}

A discrete element method (DEM), also called a distinct element method originally developed by Cundall and Strack is any of family of numerical methods for computing the motion and effect of a large number of small particles. It has been applied in many fields including process engineering, mining, and Geomechanics. It is also recognized as an effective method for the study of the fundamental behaviour of granular materials $[1,2]$.

There are a number of commercial simulation packages and open source software available to perform DEM modelling. In this study LIGGGHTS is used to carry out the DEM simulation [6].

\section{Material Properties}

Some important parameters used in this study with significant effects on the macroscopic properties of the fine granular material are: particle-particle sliding and rolling friction coefficients, particle density and particle diameter. In this study, the pile is made of fine sand and its characteristic [7] is presented in table 1 .

TABLE 1. The sand propertied used in the simulations

\begin{tabular}{lcc}
\hline \multicolumn{1}{c}{ Variable Name } & Symbol & Value \\
\hline Particle Radius & $\mathrm{r}(\mathrm{mm})$ & 0.25 \\
Rolling Friction Coefficients & $\mu_{r . p p}$ & 0.05 \\
Sliding Friction Coefficients & $\mu_{s . v p}$ & 0.50 \\
Poisson Ratio & $\nu$ & 0.3 \\
Young's Modulus & $\mathrm{E}(\mathrm{N} / \mathrm{m} 2)$ & $50 \mathrm{e} 6$ \\
Coefficient of Restitution & $\mathrm{e}$ & 0.8 \\
\hline
\end{tabular}

\section{DEM Time Step}

The Time-Step is periodically calculated based on estimations of the Rayleigh- and Hertz time during the simulation. Time-Step has to be a fraction $(10 \%)$ of the Rayleigh time [8]:

$\operatorname{Tr}=\pi r \sqrt{(\rho / G)} /(0.163 v+0.8766)$
$\operatorname{Th}=2.87 \times\left(m^{2} /\left(r \times E^{2} \times V_{\text {max }}\right)\right)^{0.2}$

Additionally, the ratio of skin size to the distance that particles can travel relative to each other at one time-step should be greater than 1, otherwise some interactions may be missed or overlap energy may be generated artificially [6].By considering the criteria of the Rayleigh and Hertz time, some initial simulations performed and its results indicated that the suitable Time-Step for DEM simulation is 5.0E-06 second.

\section{Computational Fluid Dynamic (CFD)}

CFD analysis was performed by OpenFOAM which is an object-oriented $\mathrm{C}++$ library of classes and routines of use for writing CFD codes. With OpenFOAM it is easy to add any modification to any part of the implementation.

\section{Initial and Boundary Conditions}

Velocity inlet boundary conditions are used to define the flow velocity in the inlet. The velocity is not constant value in the inlet and is following the power law relationship. The profile of the power law relationship is[5]:

$U / U_{0}=(z / \delta)^{\alpha} u$

where $\mathrm{u}$ is the longitudinal wind velocity component and $\mathrm{U}_{0}$ is the known wind speed at boundary layer thickness $(\delta=0.1 \mathrm{~m})$. The exponent $\left(\alpha_{\mathrm{u}}\right)$ is an empirically derived coefficient that varies depending on the stability of the fluid flow. Experimental results of Farimani et al. [5] proposed 0.11 for $\alpha_{\mathrm{u}}$. This new boundary condition is named 'powerLaw' and compiled as a new dynamic library in OpenFoam 2.1.

To reduce the effect of the inlet and outlet on the flow field, the pile is placed at an extended distance from these boundaries. While OpenFOAM always generates geometries in 3 dimensions, it can be instructed to solve in 2 dimensions by specifying a special empty condition on front and back walls whose planes are normal to the 3rd dimension. These walls are defined as a periodic boundary condition in the LIGGGHTS.

The dimensions of the domain are parametrically specified and are shown in figure 1 .

\section{Turbulence Modelling}

The standard k- $\varepsilon$ model is a model based on model transport equations for the turbulence kinetic energy $(\mathrm{k})$ and its dissipation rate $(\varepsilon)$ and is used to model turbulence.

In the derivation of the $\mathrm{k}-\varepsilon$ model, the assumption is that the flow is fully turbulent, and the effects of molecular viscosity are negligible. The standard k$\varepsilon$ model is therefore valid only for fully turbulent flows [9]. 


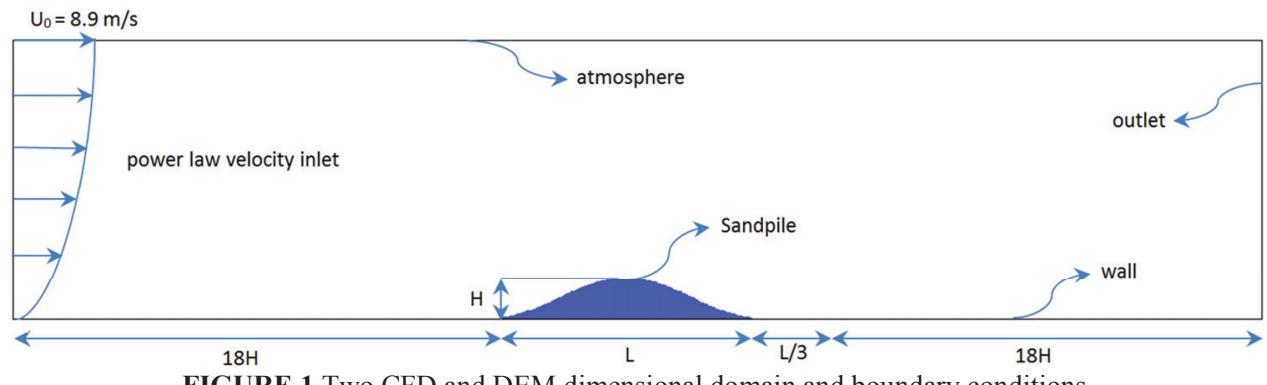

FIGURE 1.Two CFD and DEM dimensional domain and boundary conditions.

\section{CFD-DEM Model}

CFD-DEM model is suitable for the modelling or simulation of fluid-solids or fluid-particles systems. In the CFD-DEM, the motion of discrete solids or particle phase is obtained by the Discrete Element Method (DEM) which applies Newton's laws of motion to every particle, while the flow of continuum fluid is described by the local averaged Navier-Stokes equations that can be solved using the traditional Computational Fluid Dynamics (CFD) approach. The CFDEM coupling is an open source CFD-DEM framework [6].

\section{Governing Equations for Coupled Flow}

The governing equations of flow solved in this study are:

$$
\frac{\partial\left(\varepsilon_{f}\right)}{\partial t}+\nabla \cdot\left(\varepsilon_{f} u\right)=0
$$

$$
\begin{aligned}
& \partial\left(\rho_{f} \varepsilon_{f} u_{f}\right) / \partial t+\nabla .\left(\rho_{f} \varepsilon_{f} u_{f} u_{f}\right)=-\varepsilon_{f} \nabla p-F_{p f}+ \\
& \varepsilon_{f} \nabla \cdot \tau+\rho_{f} \varepsilon_{f} g
\end{aligned}
$$

where $F_{p f}$ is the volumetric particle-fluid interaction force. This force is made up of the particle drag force, the pressure gradient force, and the viscous force due to fluid shear stress. The interaction term between the air and the particles, i.e., the drag force in the momentum equation, is calculated based on the formula by Di Felice[10].

\section{RESULTS AND DISCUSSION}

\section{Grid Size}

The computing of physical values of the continuous phase in each fluid cell is achieved by applying iterative methods to the discretised conservation equations. Almost alliterative solution methods converge on finer grids with a higher accuracy, but require considerably more computing time[11]. Three different mesh size models were developed and analysed, including coarse mesh, medium mesh, and fine mesh. The CFD cell size approximately is $4 \mathrm{~mm}, 1 \mathrm{~mm}$ and $0.5 \mathrm{~mm}$ for coarse, medium, and fine mesh, respectively. The CFDEM results illustrate that the deformation of sand pile shape for only fine mesh is close to the experimental data and medium and coarse mesh are not able to simulate the erosion and the profile deformation of the sand pile. A more increment in the CFD cell size causes more particles locate in a cell. As the pressure quantity is similar inside of each cell therefore the pressure distribution around the particles in the same cell will be equal. It means the fluid flow only can be sensed in the boundaries of the cells.

Figure 2 shows the pressure distribution around sand pile and it can be seen pressure difference around a cell is really insignificant (less than $0.05 \mathrm{~N} / \mathrm{m}^{2}$, cell size $=4 \mathrm{~mm})$.

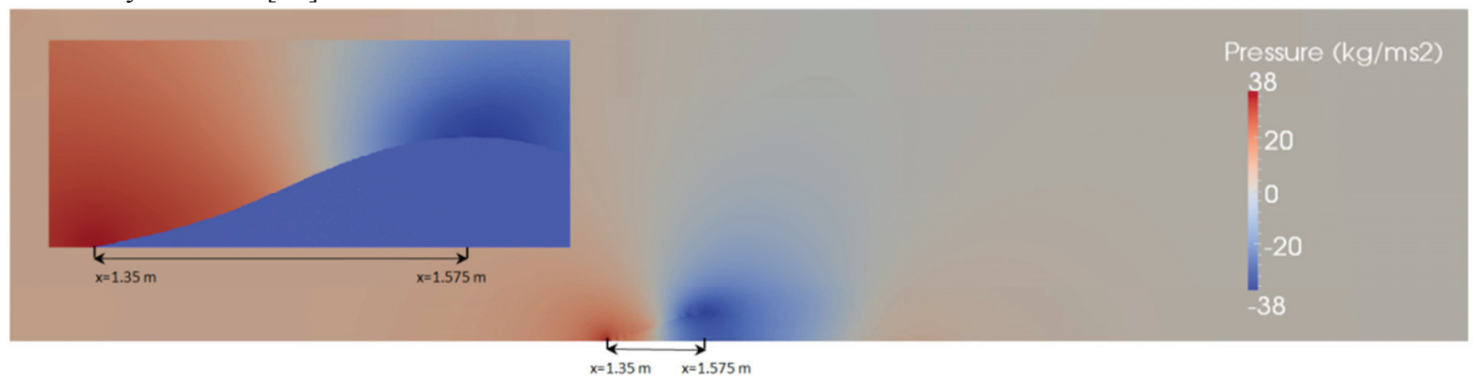

FIGURE 2. Pressure domain around sand pile after 30 second $\left(\mathrm{U}_{0}=8.9 \mathrm{~m} / \mathrm{s}\right)$. 
Simulation results confirm that the pressure distribution around one cell is not large enough to move that number of particles. Thus the fine cell in the order of particle radius is recommended to be used for simulating sand pile deformation.

\section{Saltation and Dust Liberation}

In geology, saltation is a specific type of particle transport by fluids such as wind or water. It occurs when loose material is removed from a bed and carried by the fluid, before being transported back to the surface. Earlier studies indicated that the wind velocity plays an important role in the rate of erosion and dust liberation[5]. An approximate number of particles that have left the sand pile surface in the first 30 seconds of simulation in the various wind velocity has been shown in the table 2 . It can be concluded that increasing the wind velocity increases nonlinearly the rate of dust liberation and speed of sand pile deformation.

TABLE 2.An approximate number of particles that have left the sand pile surface after 30 seconds of simulation

\begin{tabular}{cc}
\hline Wind velocity & Number of particles \\
\hline $5(\mathrm{~m} / \mathrm{s})$ & 1200 \\
$8.9(\mathrm{~m} / \mathrm{s})$ & 5000 \\
$12(\mathrm{~m} / \mathrm{s})$ & 8500 \\
\hline
\end{tabular}

Figure 3 shows sand pile deformation as a result of saltation and creep while the wind speed was $8.9 \mathrm{~m} / \mathrm{s}$. Farimani et al. validated their CFD simulation with the experimental results.

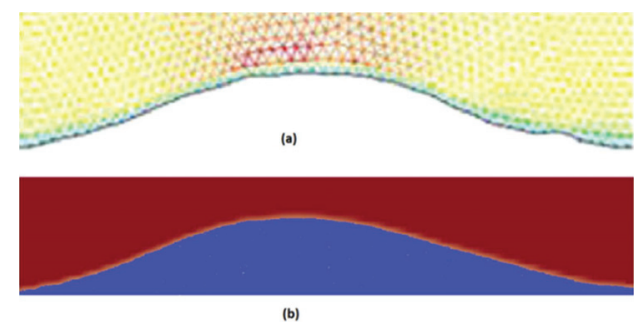

FIGURE 3.Comparison between sand pile deformation after 30 second (a : Farimani et al. [5], b: this study)

The first available experimental result is for $t=120$ second therefore CFD-DEM results were only compared with the $\mathrm{CFD}$ results at $\mathrm{t}=30$ second. It can be seen CFD-DEM coupling results are in a good agreement with the simulation results of Farimani et al. [5].

\section{CONCLUSION}

In these study results of CFD-DEM modelling is extracted from the first 30 second of the erosion process. Stockpile deformation was simulated and finally the accuracy of stockpile deformation results obtained by CFD-DEM modelling was compared with the experimental results of Farimani et al [5].

Simulation results show that the pressure distribution around one cell should be large enough to be able to move the particles. The size of CFD cells is suggested to be in the order of particle radius for this kind of simulation. It was seen that wind velocity has a significant effect on the rate of dust liberation. In the other word, dust liberation rate increases nonlinearly with increasing wind speed. It was shown that coupling CFD-DEM can be used as a possible method to illustrate erosion and deformation of stockpiles.

\section{REFERENCES}

[1] A.D. Ferreira, R.A. Oliveira, Journal of Wind Engineering and Industrial Aerodynamics 1, 1-10 (2009).

[2] I. Diego, A. Pelegry, S. Torno, J. a. Toraño, M. Menendez, Applied Mathematical Modelling 333197 3207 (2009).

[3] T. Badr, J.-L. Harion, Atmospheric Environment 41, 360-368 (2007).

[4] C. Turpin, J.L. Harion, Atmospheric Environment 43, 5579-5587 (2009).

[5] A.B. Farimani, A.D. Ferreira, A.C.M. Sousa, Geomorphology 130, 299-311 (2011).

[6] C. Kloss, C. Goniva, A. Hager, S. Amberger, S. Pirker, Progress in Computational Fluid Dynamics 12, 140 152 (2012).

[7] S.M. Derakhshani, D.L. Schott, G. Lodewijks, Reducing computational time by adjusting domain frame for fine granular materials with Discrete Element Method, in: Bulksolids Europe, Berlin, Germany, 2012: pp. 0-7.

[8] C. Kloss, S. Pirker, Coupling of DEM and CFD Simulation and Experiment, in: Discrete Element Method, Kopenhagen, n.d.

[9] V. Yakhot, S.A. Orszag, Journal of Scientific Computing 1, 3-51 (1986).

[10] R. Di Felice, International Journal of Multiphase Flow 20, 153-159(1994).

[11] F. Alobaid, J. Ströhle, B. Epple, Advanced Powder Technology 24, 403-415 (2012). 